03.1

\title{
Трансформация отрывного турбулентного течения в конической лунке на стенке узкого канала и снижение гидравлических потерь с увеличением конусности
}

\author{
(C) С.А. Исаев ${ }^{1,2}$, Д.В. Никущенко ${ }^{1}$, А.Г. Судаков ${ }^{2}$, Н.В. Тряскин ${ }^{1}$, Л.П. Юнаков ${ }^{3}$ \\ ${ }^{1}$ Санкт-Петербургский государственный морской технический университет, Санкт-Петербург, Россия \\ ${ }^{2}$ Санкт-Петербургский государственный университет гражданской авиации, Санкт-Петербург, Россия \\ ${ }^{3}$ Балтийский государственный технический университет, Санкт-Петербург, Россия \\ E-mail: isaev3612@yandex.ru
}

Поступило в Редакцию 18 января 2021г.

В окончательной редакции 27 февраля 2021 г.

Принято к публикации 6 марта 2021 г.

На основе решения нестационарных осредненных по Рейнольдсу уравнений Навье-Стокса (URANS), замкнутых с помощью модифицированной модели переноса сдвиговых напряжений (SST), рассчитано отрывное течение в узком канале с конической лункой на стенке с углом уклона $\theta$. Отмечено, что наблюдается резкий переход от периодического режима с двумя попеременно изменяющимися вихрями в близких к цилиндрическим $\left(\theta=10-22.5^{\circ}\right)$ лунках к режимам с периодическими низкочастотными колебаниями наклонных моносмерчевых структур при умеренных $\theta\left(30-37.5^{\circ}\right)$ и с установившимися вихревыми структурами при $\theta$ свыше $45^{\circ}$. Относительные гидравлические потери на участке канала с лункой при увеличении $\theta$ остаются на уровне $1.23-1.24$ до $\theta=45^{\circ}$, а затем быстро снижаются почти в 1.5 раза до 1.16 при $\theta=60^{\circ}$.

Ключевые слова: конические лунки, узкий канал, интенсификация отрывного течения.

DOI: 10.21883/PJTF.2021.11.51002.18714

Энергоэффективные поверхности, структурированные упорядоченными лунками [1], активно внедряются в вихревые технологии для энергетики. На их основе можно обеспечить опережающий рост тепловой эффективности по сравнению с возрастанием гидравлических потерь. Наиболее распространены сферические лунки. Исследования цилиндрических и конических лунок не столь многочисленны и не носят систематического характера. Так, в [2] наблюдались парные вихри в цилиндрической лунке с относительной глубиной $h / D<0.2$ $(D-$ диаметр пятна) и флуктуации пространственного течения в глубокой лунке с $h / D$ порядка 0.4-0.5. При обтекании цилиндрического углубления (0.1) на стенке гидроканала в [3] обнаружено, что парные вихри изменяют направление вращения на обратное при числе Рейнольдса $\operatorname{Re}_{D}>12000$. В исследовании [4] теплообмена в узком канале с конической лункой глубиной $h / D=0.233$ при $\operatorname{Re}_{D}=40000$ установлено, что по мере трансформации формы лунки от усеченного конуса к цилиндрической поверхности отрывное течение в ней становится более интенсивным и возрастает теплоотдача от ареала с лункой. В настоящей работе уточняется физический механизм интенсификации отрывного течения в такой конической лунке при варьировании угла уклона $\theta$ от 10 до 60․ Также проводится сравнение со сферической лункой близкой глубины $h / D=0.26$.

Обтеканию конических лунок с относительной глубиной 0.233 присуща бифуркация вихревых структур, обнаруженная в сферических лунках на стенке канала при увеличении их глубины [5]. В лунках одинаковой глубины $(h / D$ порядка 0.2$)$ в зависимости от предыстории течения могут возникать режимы с образованием симметричных парных вихрей и моносмерчевой наклонной структуры. Для конических лунок также характерен периодический режим с двумя попеременно изменяющимися вихрями, напоминающий реализующийся в глубоких сферических [6,7] лунках переключательный режим и связанный с периодическим изменением сгенерированных на боковых склонах смерчеобразных структур.

Рассматривается конфигурация узкого плоскопараллельного канала с конической лункой на нижней стенке (рис. 1), апробированная в ряде исследований $[8,9]$. Она является компьютерным аналогом канала экспериментальных стендов (в частности, в [6,7]). В качестве характерных параметров выбираются диаметр пятна лунки $D$, среднемассовая скорость потока $U$ и отношение $D / U$ (единица времени). Высота канала принимается равной 0.33 , а ширина канала - 2.5. Степень турбулентности задается близкой к экспериментальной $(1-5 \%)$, а масштаб турбулентности принимается порядка характерного размера. Относительный радиус скругления кромки 0.025. Расчетная область охватывает ограниченное стенками пространство узкого канала протяженностью 6.7 (рис. 1). Входная проточная граница с профилями характеристик полностью развитого турбулентного потока располагается на расстоянии 1.4 от проекции 


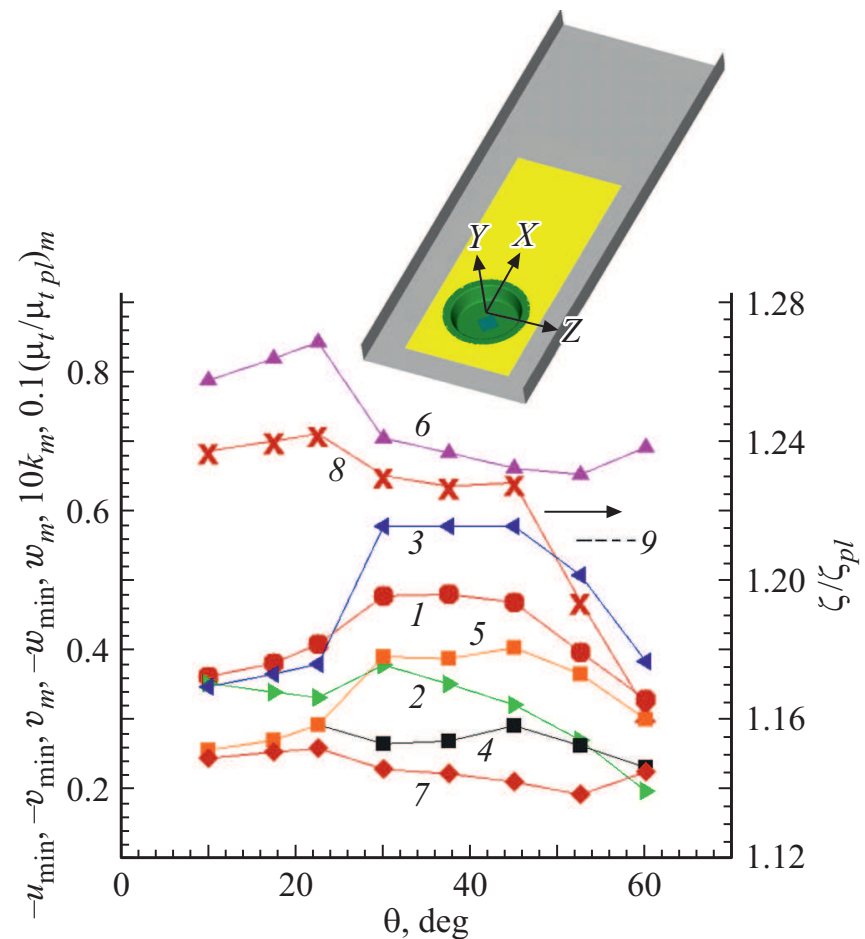

Рис. 1. Зависимости экстремальных параметров течения $(1-5)$, характеристик турбулентности $(6,7)$ и гидравлических потерь на участке канала с конической $(8)$ и сферической $(9)$ лунками от угла уклона $\theta:-u_{\min }(1),-v_{\min }(2), v_{m}(3),-w_{\min }$ (4), $w_{m}(5), 10 k_{m}(6),\left(\mu_{t} / \mu_{t p l}\right)_{m}(7), \zeta / \xi_{p l}(8,9)$.

центра лунки на плоскость нижней стенки, принимаемой в качестве центра декартовой системы координат $X, Y, Z$. В выходном сечении канала задаются „мягкие“ граничные условия - условия продолжения решения на границу [8,9], а на омываемых стенках ставятся условия прилипания.

Численное решение нестационарных осредненных по Рейнольдсу уравнений Навье-Стокса (URANS), замкнутых с помощью модифицированной SST-модели турбулентности $[5,8,9]$, базируется на концепции расщепления по физическим процессам, использует процедуру коррекции давления SIMPLEC $[8,9]$ и многоблочные структурированные сетки с частичным перекрытием. Используется метод второго порядка аппроксимации по пространственным переменным и первого порядка по времени. Безразмерный шаг по времени 0.02. Вычислительный процесс заканчивается при выходе на периодические режимы изменения локальных и интегральных параметров, включая поперечную силу $R_{z}$, действующую на контрольный участок с лункой размером $2.5 \times 1.5$ со смещением центра вниз по потоку на 0.5 . Приемлемость URANS для расчета пространственных отрывных течений обосновывается в ряде численных исследований, в том числе в расчетах обтекания кубика в канале [10]. В настоящей работе анализируются осредненные по периоду колебаний локальные и интегральные характеристики по методике [11]. В их число входят распределения перепада коэффициентов давления на омываемой стенке с лункой и стенке плоскопараллельного канала $C_{p}-C_{p p l}$ и относительного трения $C_{f} / C_{f p l}$ в срединном продольном сечении лунки. Также анализируются зависимости от угла уклона $\theta$ экстремальных характеристик течения $\left(u_{\min }\right.$, $\left.v_{\min }, v_{m}, w_{\min }, w_{m}\right)$, турбулентности $\left(k_{m}, \mu_{t} / \mu_{t p l}\right)$ и относительных коэффициентов гидравлических потерь $\left(\xi / \xi_{p l}\right)$. Параметры с индексом $p l$ получены при расчете течения в аналогичном канале без лунки. Величина $\xi$ определяется на основе расчета перепада полного давления между поперечными сечениями канала по методике [8].

На рис. 1-3 и в таблице представлены некоторые из полученных результатов.

Нестационарное обтекание лунок на стенке узкого канала наблюдается в диапазоне изменения $\theta$ от 10 до $37.5^{\circ}$. До $\theta=22.5^{\circ}$ течение внутри конической лунки носит периодический характер, причем период низкочастотных колебаний поперечной силы $R_{z}$ достаточно велик (число Струхаля Sh порядка 0.01-0.02). Для конических лунок с углами уклона $\theta=30$ и $37.5^{\circ}$ формируется другой тип турбулентного течения с периодом колебаний на порядок меньше (Sh имеет величину 0.25-0.33), чем для лунок с малыми углами уклона. Струйно-вихревые структуры, реализующиеся в лунках, медленно флуктуируют, оставаясь наклоненными под углами $\pm 45^{\circ}$ к набегающему потоку. Осредненные по периоду колебаний картины растекания воздуха по облуненной стенке (рис. 2) резко изменяются от симметричной картины линий тока, нанесенных на поля давления $\left(\theta=22.5^{\circ}\right)$, к несимметричной (при $\left.\theta=30^{\circ}\right)$. При углах $\theta$ свыше $45^{\circ}$ течение около конической лунки на стенке узкого канала оказывается стационарным и сохраняет устойчивую асимметричную вихревую структуру.

Как видно из рис. 1, наибольшая величина скорости возвратного течения в лунке $\left(-u_{\min }\right)$ значительно увеличивается по мере уменьшения угла $\theta$ : от 0.33 при $\theta=60^{\circ}$ до 0.48 при $\theta=37.5^{\circ}$. На этом уровне она остается до наступления режима с двумя вихрями, при котором резко падает до 0.4 и далее медленно снижается до 0.36 при $\theta=10^{\circ}$. Наибольшая скорость вторичного течения $w_{m}$ также возрастает от 0.3 до 0.4 по мере уменьшения $\theta$ с 60 до $45^{\circ}$. Далее $w_{m}$ изменяется мало (в пределах 0.01), а в режиме с двумя вихрями наблюдается резкое снижение: до 0.3 при $\theta=22.5^{\circ}$ с последующим медленным уменьшением до 0.25 при $\theta=10^{\circ}$. Наиболее значительные изменения наблюдают ся в наибольших величинах скоростей восходящих $\left(v_{m}\right)$ потоков. Медленное возрастание $v_{m}$ в режиме с двумя вихрями по мере увеличения $\theta$ сменяется скачкообразным ростом от 0.4 до 0.58 при переходе к режиму с образованием наклонного моносмерчевого вихря. Эта довольно значительная величина $v_{m}$ остается неизменной в промежутке изменения углов $\theta$ от 30 до $45^{\circ}$. Затем она быстро 
$a$

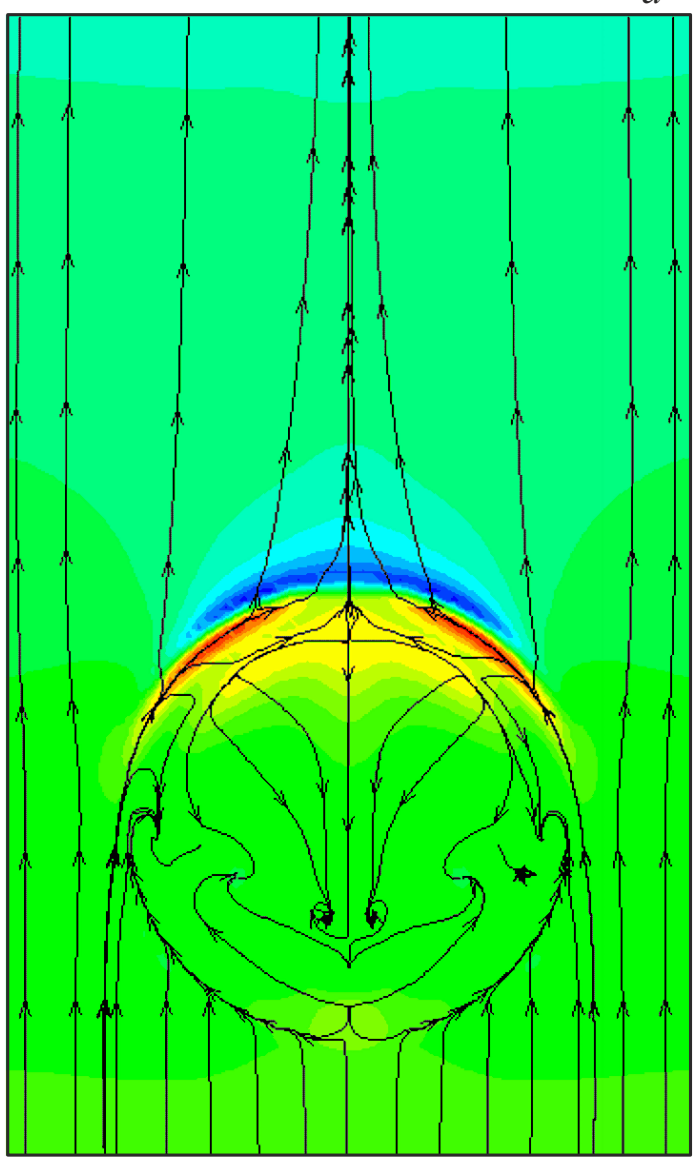

$b$
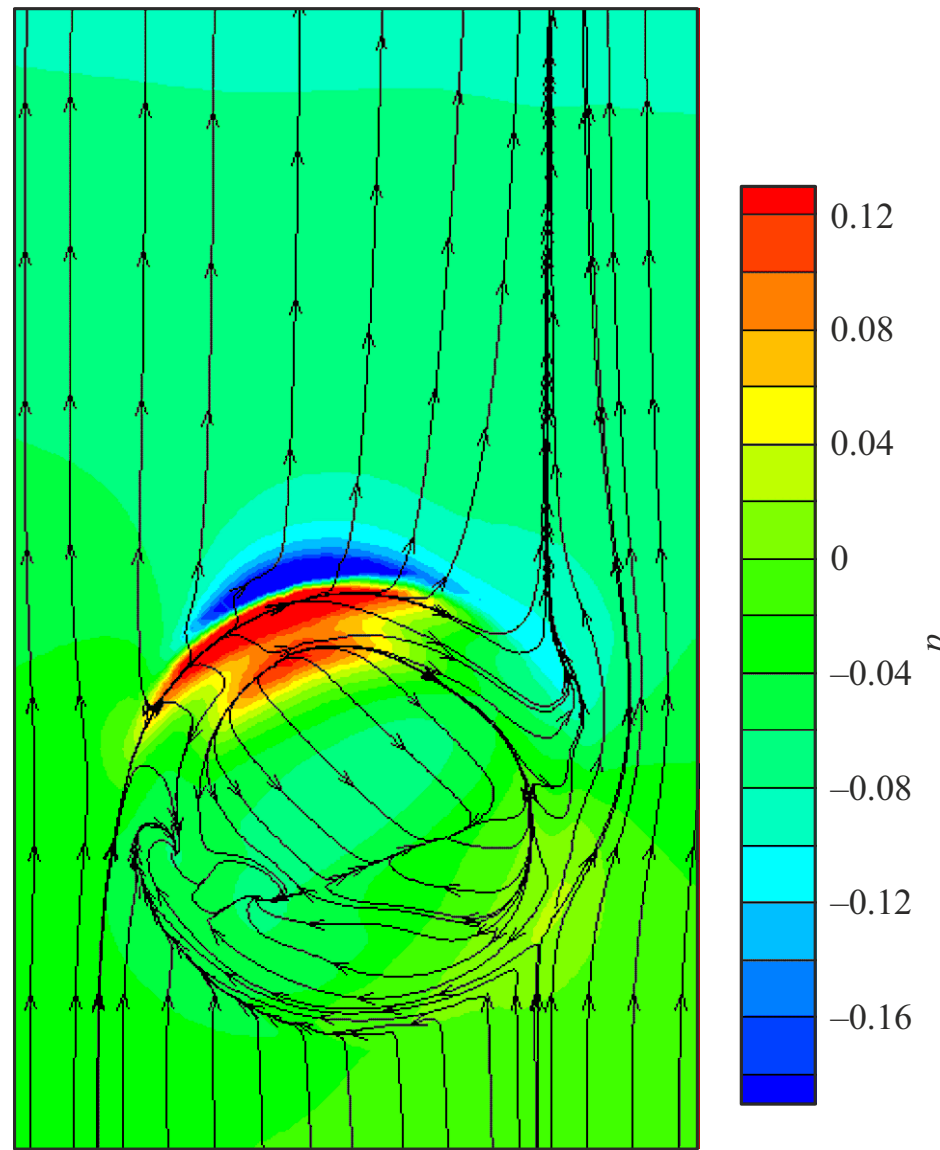

Рис. 2. Сравнение полей давления с нанесенными линиями растекания воздуха по облуненной стенке для лунок с $\theta=22.5(a)$ и $30^{\circ}(b)$.

снижается до 0.38 при $\theta=60^{\circ}$. Увеличение $\theta$ в режиме с двумя вихрями немного повышает уровень максимальных величин турбулентной энергии (до 0.084). Далее для режима обтекания конических лунок с асимметричными вихревыми структурами $k_{m}$ заметно ниже и изменяется в диапазоне от 0.065 до 0.07. Максимальная величина относительной вихревой вязкости находится в диапазоне 2-2.6, причем наибольшие значения приходятся на лунки с малыми углами уклона. Относительные гидравлические потери участка с лункой резко нарастают от 1.165 до 1.227 по мере уменьшения угла $\theta$ от 60 до $45^{\circ}$. Далее они слабо меняются до наступления режима с двумя вихрями и не превышают 1.23 . С ростом $\theta$ от 10 до $22.5^{\circ} \xi / \xi_{p l}$ медленно нарастают от 1.237 до 1.242 , т.е. заметно превосходят все остальные значения. Интересно отметить, что гидравлические потери на участке канала со сферической лункой оказываются порядка 1.21 и превосходят $\xi / \xi_{p l}$ для конической лунки при $\theta=52.5^{\circ}$.

Как уже отмечалось, осреднение полей скорости и давления для лунок с малыми углами уклона (рис. $2, a$ ) продуцирует симметричную картину растекания воздуха по облуненной стенке канала при $\theta=22.5^{\circ}$. Переход к асимметричной картине на рис. $2, b$ для $\theta=30^{\circ}$ показывает, что только один центр генерации закрученного потока остается на левой стороне лунки, а другой центр пропадает. Возвратное и вторичное течение в лунке заметно интенсифицируются.

Диагностика в продольном срединном сечении лунки распределений превышения коэффициента статического давления на облуненной поверхности узкого канала над коэффициентом давления на стенке плоскопараллельного канала $C_{p}-C_{p p l}$ (рис. $\left.3, a\right)$ и относительного трения $C_{f} / C_{f p l}$ (рис. $3, b$ ) показывает резкое нарастание пиковых локальных нагрузок на дно и наветренный склон лунки, а также интенсификацию отрывного течения при переходе от режима с двумя вихрями к формированию моносмерчевого вихря в конической лунке. Максимумы статического давления увеличиваются в $1.5-2$ раза, причем наибольшая величина перепада коэффициентов давления, равная 0.46 , получается при $\theta=45^{\circ}$. В угловых зонах на дне лунок наблюдаются вторичные пики локальных нагрузок, смещающиеся к центру лунки и уменьшающиеся по величине по мере 

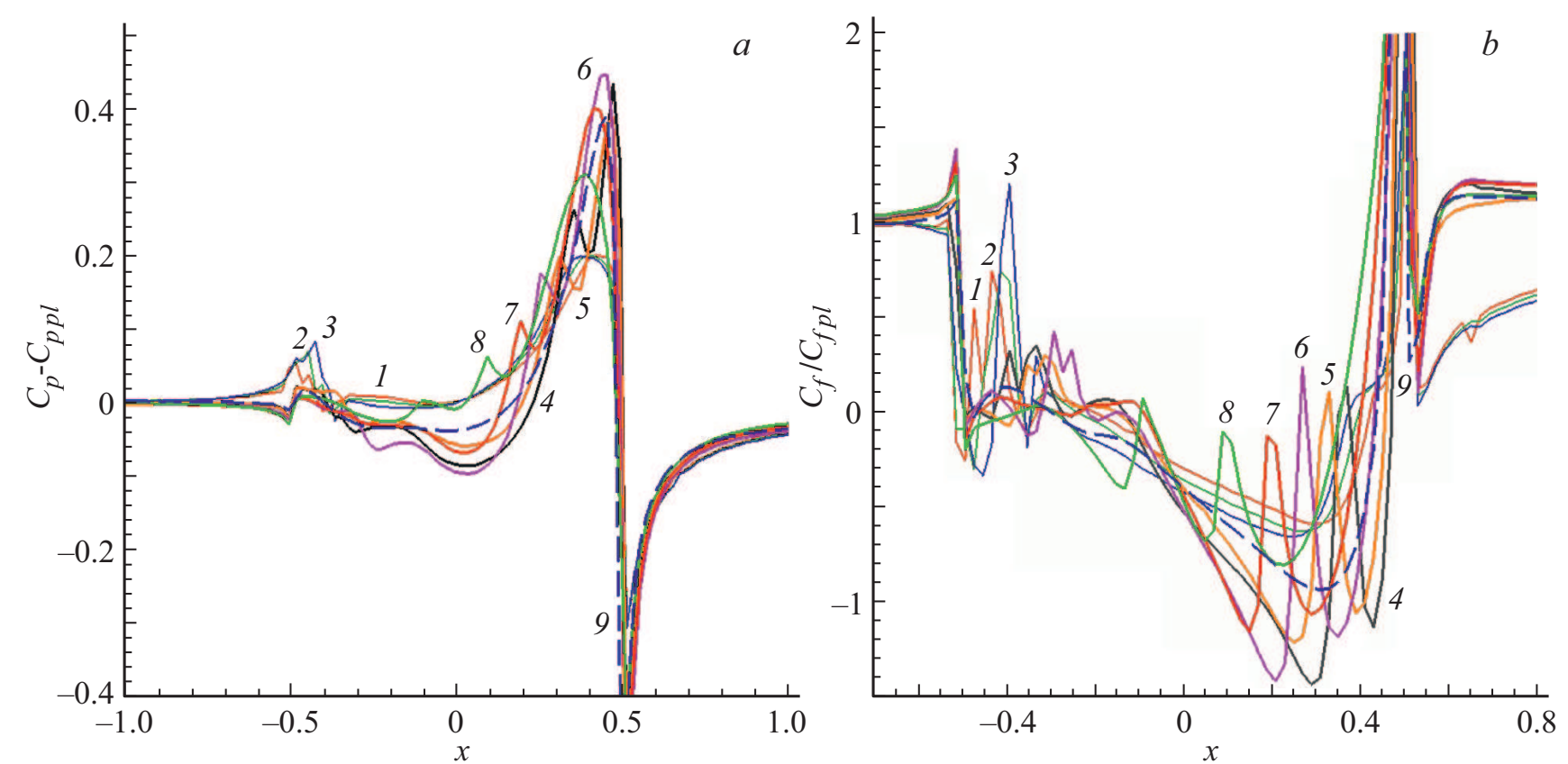

Рис. 3. Зависимости $C_{p}-C_{p p l}(a)$ и $C_{f} / C_{f p l}(b)$ в срединном продольном сечении облуненной стенки от угла уклона конической лунки $\theta . \theta=10(1), 17.5(2), 22.5(3), 30(4), 37.5(5), 45(6), 52.5(7), 60^{\circ}(8) ; 9-$ сферическая лунка.

Периоды колебаний отрывного течения в узком канале с конической лункой при различных углах уклона

\begin{tabular}{l|c}
\hline$\theta,^{\circ}$ & $T$ \\
\hline 10 & 53 \\
17.5 & 53 \\
22.5 & 72 \\
30 & 2.9 \\
37.5 & 4
\end{tabular}

увеличения угла уклона. Относительное отрицательное трение при 30 и $45^{\circ}$ достигает значения -1.4 , которое более чем в 2 раза отличается от $\left(C_{f} / C_{f} p l\right)_{\min }$ для лунок с малыми углами уклона. Для рассматриваемой сферической лунки интенсификация отрывного течения получается меньше: $\left(C_{f} / C_{f} p l\right)_{\min }$ порядка -0.9 .

Таким образом, анализируется интересный физический эффект при турбулентном обтекании глубокой конической лунки на стенке узкого канала, который характеризуется резким переходом от периодического режима с двумя вихрями к формированию моносмерчевой структуры с наклонной закрученной струей при изменении угла уклона $\theta$ от 22.5 до $30^{\circ}$. Смена режимов сопровождается интенсификацией возвратного и вторичного течения в лунке. Для $\theta$ свыше $45^{\circ}$ стабилизированное отрывное течение заметно ослабевает и гидравлические потери на участке канала с лункой существенно снижаются.

\section{Финансирование работы}

Исследования выполнены при финансовой поддержке Российского научного фонда (грант 19-19-00259).

\section{Конфликт интересов}

Авторы заявляют, что у них нет конфликта интересов.

\section{Список литературы}

[1] S. Rashidi, F. Hormozi, B. Sundén, O. Mahian, Appl. Energy, 250, 1491 (2019). https://doi.org/10.1016/j.apenergy.2019.04.168

[2] M. Hiwada, T. Kawamura, J. Mabuchi, M. Kumada, Bull. JSME, 26 (220), 1744 (1983).

[3] A.A. Khalatov, A. Byerley, S.-K. Min, D. Ochoa, ASME Paper N GT2004-53656 (2004).

[4] A.I. Leontiev, S.A. Isaev, N.V. Kornev, Ya. Chudnovsky, E. Hassel, in Proc. of the 14th Int. Heat Transfer Conf. (IHTC 14) (Washington, USA, 2010), p. 419. https://doi.org/10.1115/IHTC14-22334

[5] С.А. Исаев, А.И. Леонтьев, Н.А. Кудрявцев, И.А. Пышный, Теплофизика высоких температур, 41 (2), 268 (2003).

[6] J. Turnow, Flow structure and heat transfer on dimpled surfaces. PhD Thesis (University of Rostock, 2011).

[7] В.И. Терехов, С.В. Калинина, Ю.М. Мшвидобадзе, Прикл. математика и техн. физика, 34 (3), 40 (1993).

[8] S.A. Isaev, N.V. Kornev, A.I. Leontiev, E. Hassel, Int. J. Heat Mass Transfer, 53 (1-3), 178 (2010). DOI: 10.1016/j.ijheatmasstransfer.2009.09.042 
[9] S.A. Isaev, A.V. Schelchkov, A.I. Leontiev, P.A. Baranov, M.E. Gulcova, Int. J. Heat and Mass Transfer, 94, 426 (2016). doi.org/10.1016/j.jheatmasstransfer.2015.11.002

[10] С.А. Исаев, Д.А. Лысенко, Инж.-физ. журн., 82 (3), 492 (2009).

[11] С.А. Исаев, П.А. Баранов, Н.А. Кудрявцев, Ю.В. Жукова, Теплофизика и аэромеханика, 12 (2), 271 (2005). 